\title{
Sarcoidosis: Challenging Diagnostic Aspects of an Old Disease
}

\author{
Paolo Spagnolo, MD, PhD, ${ }^{a, b}$ Fabrizio Luppi, MD, PhD, ${ }^{a, b}$ Pietro Roversi, MD, ${ }^{a, b}$ Stefania Cerri, MD, PhD, ${ }^{a, b}$ \\ Leonardo Michele Fabbri, MD, ${ }^{a}$ Luca Richeldi, MD, PhD ${ }^{a, b}$ \\ ${ }^{a}$ Section of Respiratory Diseases, ${ }^{b}$ Center for Rare Lung Diseases, Department of Oncology, Hematology, and Respiratory Diseases, \\ University of Modena and Reggio Emilia, Modena, Italy.
}

\begin{abstract}
Over the past few years, there have been substantial advances in our understanding of sarcoidosis immunopathogenesis. Conversely, the etiology of the disease remains obscure for a number of reasons, including heterogeneity of clinical manifestations, often overlapping with other disorders, and insensitive and nonspecific diagnostic tests. While no cause has been definitely confirmed, there is increasing evidence that one or more infectious agents may cause the disease, although the organism may no longer be viable. Here we present 2 cases, in which sarcoidosis preceded tuberculosis and non-Hodgkin lymphoma. Development of new lesions in a patient with chronic/remitting sarcoidosis should be looked at with suspicion and promptly investigated in order to rule out an alternative/concomitant diagnosis. In such cases, tissue confirmation from the most accessible site, and bone marrow biopsy-if lymphoma is in the differential diagnosis - should be performed. In conclusion, we strongly advise that physicians be ready to reconsider the diagnosis of sarcoidosis in the presence of atypical manifestations or persistent/progressive disease despite conventional therapy.

(C) 2012 Elsevier Inc. All rights reserved. - The American Journal of Medicine (2012) 125, 118-125
\end{abstract}

KEYWORDS: Granuloma; Lymphoma; Mycobacterium tuberculosis; Sarcoidosis

When a thing ceases to be a subject of controversy, it ceases to be a subject of interest.

\section{William Hazlitt}

Sarcoidosis is a systemic inflammatory disorder of unknown etiology, characterized pathologically by noncaseating epithelioid cell granulomas-primarily affecting the lungs and the lymphatics - and clinically by variable manifestations and course. While remission, with or without treatment, occurs in the majority of cases, sarcoidosis may persist as chronic disease in up to one third of patients, causing significant morbidity. ${ }^{1}$ Currently, the consensus

\section{Funding: None.}

Conflict of Interest: None.

Authorship: All authors had access to the data and a role in writing the manuscript.

Requests for reprints should be addressed to Paolo Spagnolo, MD, $\mathrm{PhD}$, Section of Respiratory Diseases and Center for Rare Lung Diseases, Department of Oncology, Hematology, and Respiratory Diseases, University of Modena and Reggio Emilia, Policlinico di Modena, via del Pozzo 71, Modena 41100, Italy.

E-mail address: paolo.spagnolo@unimore.it view is that sarcoidosis is triggered by persistent presentation of as-yet unidentified, poorly degradable antigen(s) in genetically susceptible hosts, thus triggering a Th1-type cellular immune response leading to granuloma formation. The diagnosis is established when compatible clinicoradiological features are supported by histological evidence of noncaseating epithelioid granulomas. ${ }^{2}$ In the absence of a known etiology, sarcoidosis remains a diagnosis of exclusion owing to the lack of definitive diagnostic blood, skin, or radiologic imaging tests specific for the disorder.

The recommended approach to all patients at presentation involves a series of routine screening tests in order to assess the severity of organ involvement (Table 1). Histological confirmation of the diagnosis should be obtained wherever possible. However, a CD4/CD8 T lymphocyte ratio higher than 3.5 in bronchoalveolar lavage fluid (BAL), angiotensin-converting enzyme (ACE) levels twice higher than the upper normal limit, typical abnormal calcium metabolism, and negative bacterial or fungal cultures may be helpful in establishing the diagnosis. ${ }^{2}$ 


\section{CLINICAL CASE 1}

A 58-year-old man, a lifelong nonsmoker, had been previously diagnosed with sarcoidosis (based on transbronchial biopsies showing epithelioid non-necrotizing granulomas and compatible clinicoradiologic features) and successfully treated with oral steroids for 6 months (Figure 1). At that time, tuberculin skin test was negative. Nine years later he presented with 3 months history of night fever (up to $39^{\circ} \mathrm{C}$ ), abdominal pain (left upper quadrant), fatigue, and weight loss. The patient was referred to our chest clinic because of lack of response to broad-spectrum antibiotics. An abdominal ultrasound showed enlarged spleen and liver with concomitant ascites. A recurrence of sarcoidosis was suspected, and the patient was started on $40 \mathrm{mg}$ daily of oral prednisolone, with rapid improvement. However, as the steroids were tapered, intermittent night fever and abdominal pain re-

\section{CLINICAL SIGNIFICANCE}

- Sarcoidosis may represent a diagnostic challenge even for expert physicians, because there is no single diagnostic test for this disease.

- The presence of noncaseating granulomas does not establish the diagnosis, because conditions such as tuberculosis and malignancies, among others, can cause granulomas.

- The diagnosis of sarcoidosis should be reconsidered in the presence of atypical manifestations or persistent/progressive disease despite appropriate therapy. curred. Subsequently, the patient developed left pleural effusion and severe pancytopenia. Both pleural fluid and bone marrow cultures were negative for acid-fast bacilli and fungal stain. As the patient continued to deteriorate, he underwent splenectomy and drainage of ascitic fluid, which revealed the presence of Mycobacterium tuberculosis (Figure 2). The patient was treated with standard antituberculosis therapy for 6 months and fully recovered. After 3 years' follow-up he is completely asymptomatic.

Table 1 Recommended Initial Evaluation of New Sarcoidosis Patients

Thorough past medical history (including family history), with emphasis on occupational and environmental exposure

Physical examination, looking for pulmonary, cutaneous, ocular, cardiac, hepatic, neurological, or splenic involvement

Chest radiography

Pulmonary function tests: spirometry with measurement of diffusing capacity of carbon monoxide

Full blood counts

Serum chemistries: calcium, hepatic, and renal function, CRP, ESR, ACE

24-hour urinary calcium excretion

ECG

Routine ophthalmologic examination

Tuberculin skin test

Other tests depending on clinical manifestations and suspicion of specific organ involvement

$\mathrm{ACE}=$ angiotensin-converting enzyme; $\mathrm{CRP}=\mathrm{C}$-reactive protein; $\mathrm{ECG}=$ electrocardiogram; $\mathrm{ESR}=$ erythrocyte sedimentation rate. infection with organisms such as Schistosoma or mycobacteria. ${ }^{6,7}$ Inorganic agents as well as organic particulates also may initiate pulmonary immune granulomas, the factor common to all being their low biodegradability and persistence, often within macrophages. ${ }^{8}$ These intriguing etiologic theories for sarcoidosis have, however, not endured. ${ }^{9}$

More traditional etiologic hypotheses point to infective/ transmissible agents, the concept being supported by the finding of granulomas in the lungs of transplants from donors known or suspected to have sarcoidosis. ${ }^{10}$ In addition, a granulomatous response can be produced by passaging pooled, filtered homogenates of mouse granulomatous tissue into other mice. ${ }^{11}$ Of note, organisms having the characteristics of M. tuberculosis were grown from some of these homogenates. However, the most persuasive evidence that sarcoidosis is a transmissible disease remains the Kveim-Siltzbach test-the intradermal inoculation of a protein extract of sarcoidosis lymph node or spleen that elicits an oligoclonal T-cell response and non-caseating granuloma formation in patients with sarcoidosis. ${ }^{12-14}$ The Kveim test is no longer used.

Since its first description, sarcoidosis has been thought to be related somehow to tuberculosis, and recent molecular and immunologic studies have revitalized interest in the causal role of mycobacteria in sarcoidosis. Using mycobacteria-specific primers and polymerase chain reaction (PCR), mycobacterial DNA has been identified in 7 of 16 sarcoidosis specimens, compared with 1 of 16 normal controls and 2 of 4 disease controls with tuberculosis. ${ }^{15}$ More recent studies using PCR analysis revealed the presence of DNA 
specific to Mycobacterium species in sarcoidosis tissues at a much higher rate than in tuberculosis-negative controls. ${ }^{16}$ Similarly, $60 \%$ of lung and lymph node tissue have been reported to contain mycobacterial DNA, most of which were classified as M. tuberculosis. ${ }^{17}$

New evidence may explain why highly sensitive molecular techniques, such as PCR, may fail to identify the genetic fingerprint of infectious agents in tissues of sarcoidosis patients. Using selective proteomic analysis of poorly soluble protein aggregates derived from affected tissues, Song and co-workers ${ }^{18}$ identified mycobacterial catalaseperoxidase $(m K a t G)$ DNA in most sarcoidosis biopsy specimens, but in none of the disease-free controls. In addition, Drake and co-workers, ${ }^{19}$ using enzyme-linked immunospot (ELISPOT) assays, detected a specific Th-1 immune response to M. tuberculosis early secretory antigenic target 6 (ESAT-6) and $K a t G$ peptides from peripheral blood mononuclear cells from sarcoidosis patients (15 of 26) and purified-protein-derivative (PPD) + subjects $(7$ of 8$)$ but not from PPD - subjects ( 1 of 24$)$, thus providing an immuno-

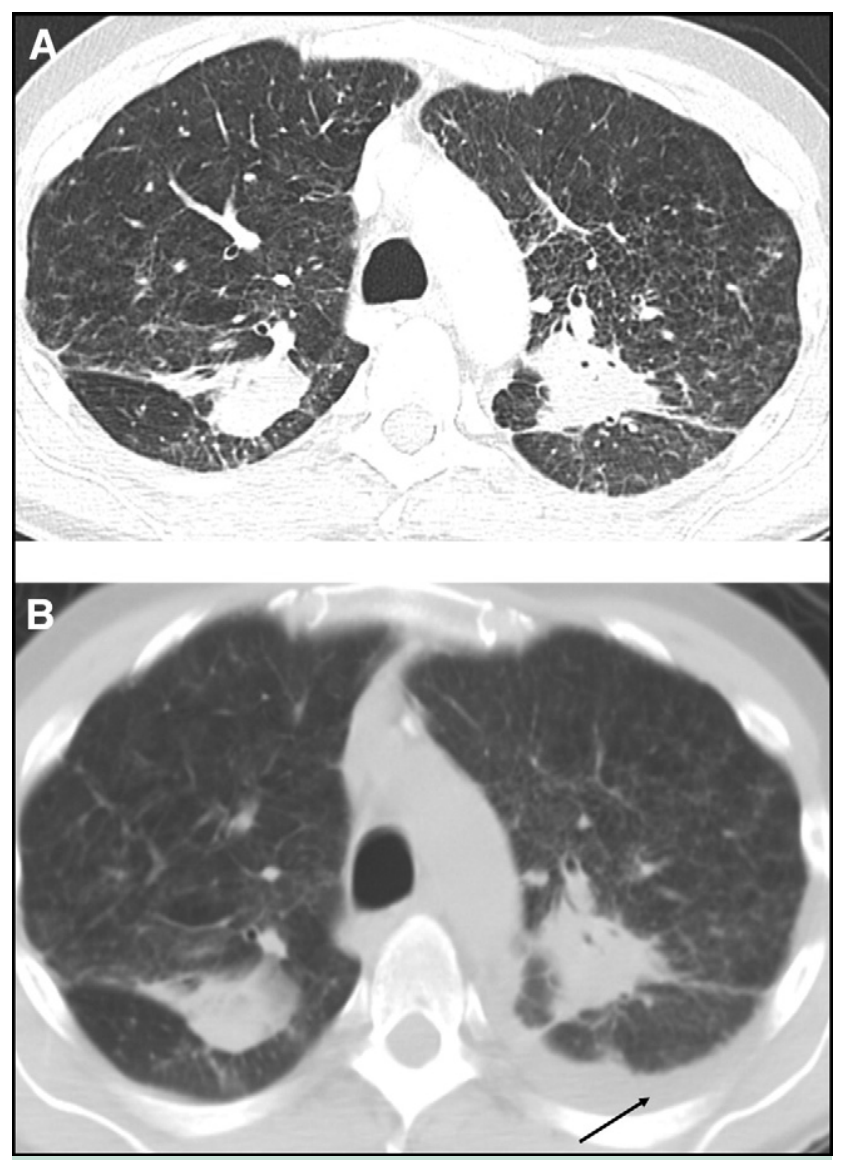

Figure 1 Computed tomography (CT) scan revealing upper lobe predominant peribronchovascular fibrotic masses. There is minimal bronchial distortion or bronchovascular deformation (A). Nine years later, the fibrotic masses appear unchanged. CT scan reveals left pleural effusion (arrow; B).

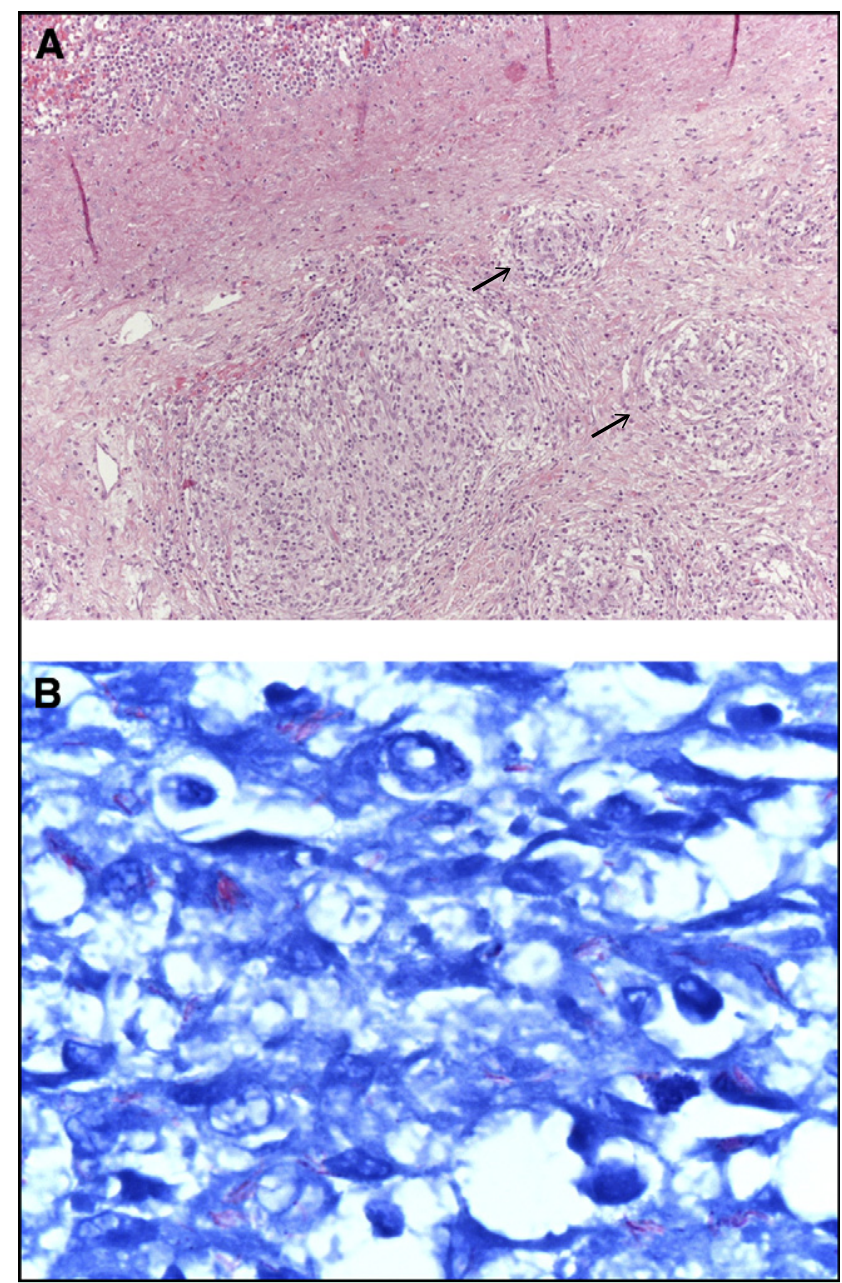

Figure 2 Tuberculous perisplenitis. Well-formed non-necrotizing granulomas (A, arrows); Mycobacterium tuberculosis (stained red) in perisplenic tissue (blue; B). A. Hematoxylineosin, $100 \times$ (courtesy Dr Alberto Cavazza). B. Ziehl-Neelsen, $1000 \times$ (courtesy Dr Alberto Cavazza).

logic link between mycobacteria and sarcoidosis. ${ }^{19}$ Furthermore, an increased BAL T-cell reactivity to $m K a t G$ has recently been shown in sarcoidosis patients, fuelling the speculation that, in at least some cases, the disease may result from an immune response initiated by mycobacteria. ${ }^{20}$

Sarcoidosis has been suspected to be an infectious disease, but no microbial pathogen has consistently been isolated or cultured so far. Alternatively, sarcoidosis may not be related to a specific exposure but rather to an abnormal immunologic host response to several agents, which would explain why many studies have come to conflicting conclusions about the cause/s of the disease. This concept, together with the wide range of its clinical manifestations, suggests that sarcoidosis may represent a family of diseases (sarcoidoses), each driven by a specific etiologic agent. ${ }^{21}$ Future studies investigating genetic risk factors could explain why some patients are found to have an association with microbial antigens and others are not. 


\section{CLINICAL CASE 2}

A 76-year-old woman, a lifelong nonsmoker, presented to our chest clinic with persistent high fever (up to $40^{\circ} \mathrm{C}$ ). She had been diagnosed 12 months earlier with a cutaneous diffuse follicular lymphoma, which did not require any systemic treatment. Chest radiograph and computed tomography $(\mathrm{CT})$ scan showed multiple enlarged mediastinal and hilar lymph nodes (Figure 3A). She underwent bronchoscopy with BAL, which revealed mild lymphocytosis (18\%) with a normal CD4/CD8 ratio, and multiple transbronchial biopsies, showing epitheliod noncaseating granulomas, thus suggestive of sarcoidosis (Figure 4). She was started on oral steroids, with rapid improvement both clinically and radiologically (Figure 3B). Eight months later, the patient again became symptomatic, complaining of persistent fever, mal-
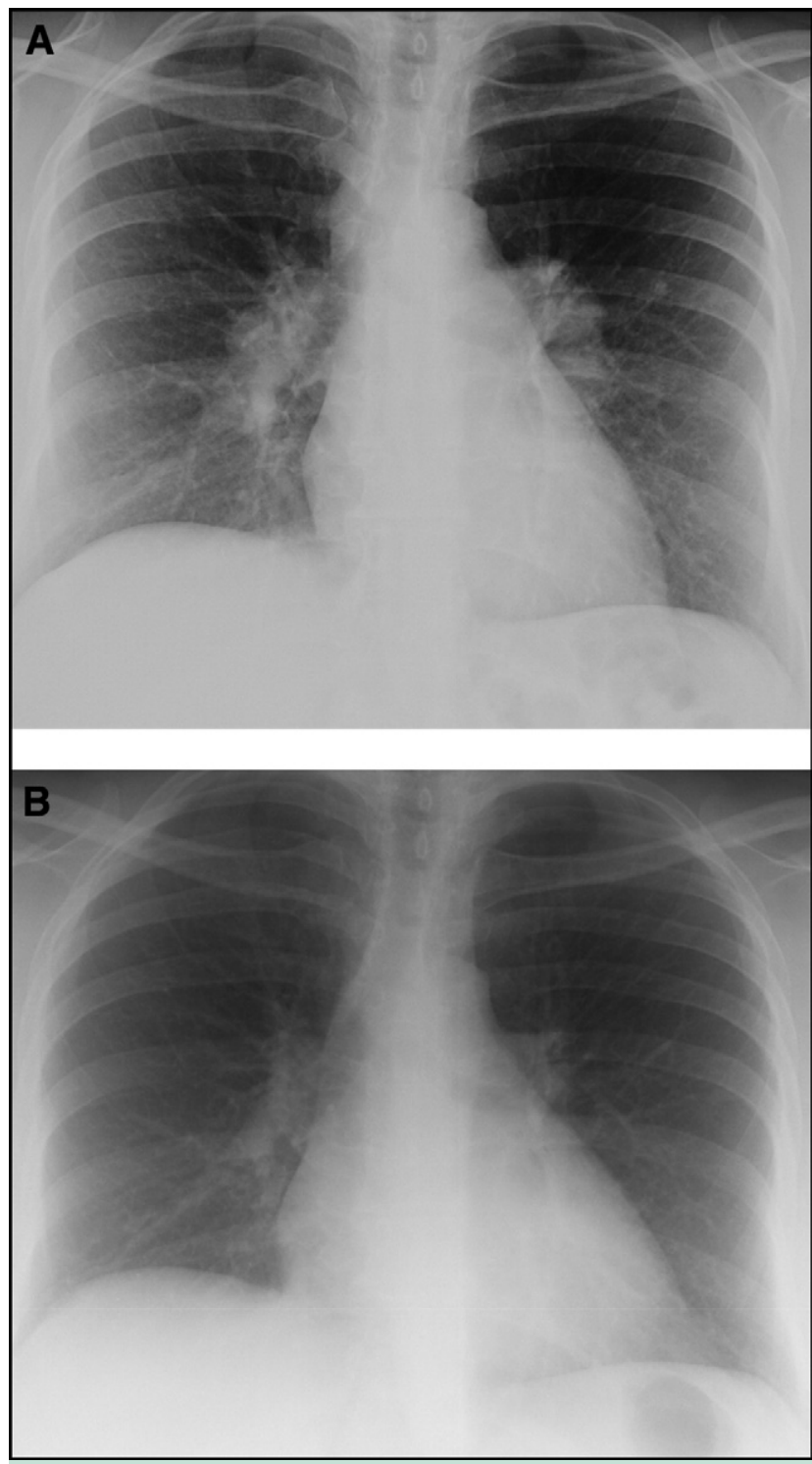

Figure 3 Chest radiograph showing bilateral hilar lymph adenopathy (A), which rapidly and completely regressed after steroid therapy (B).
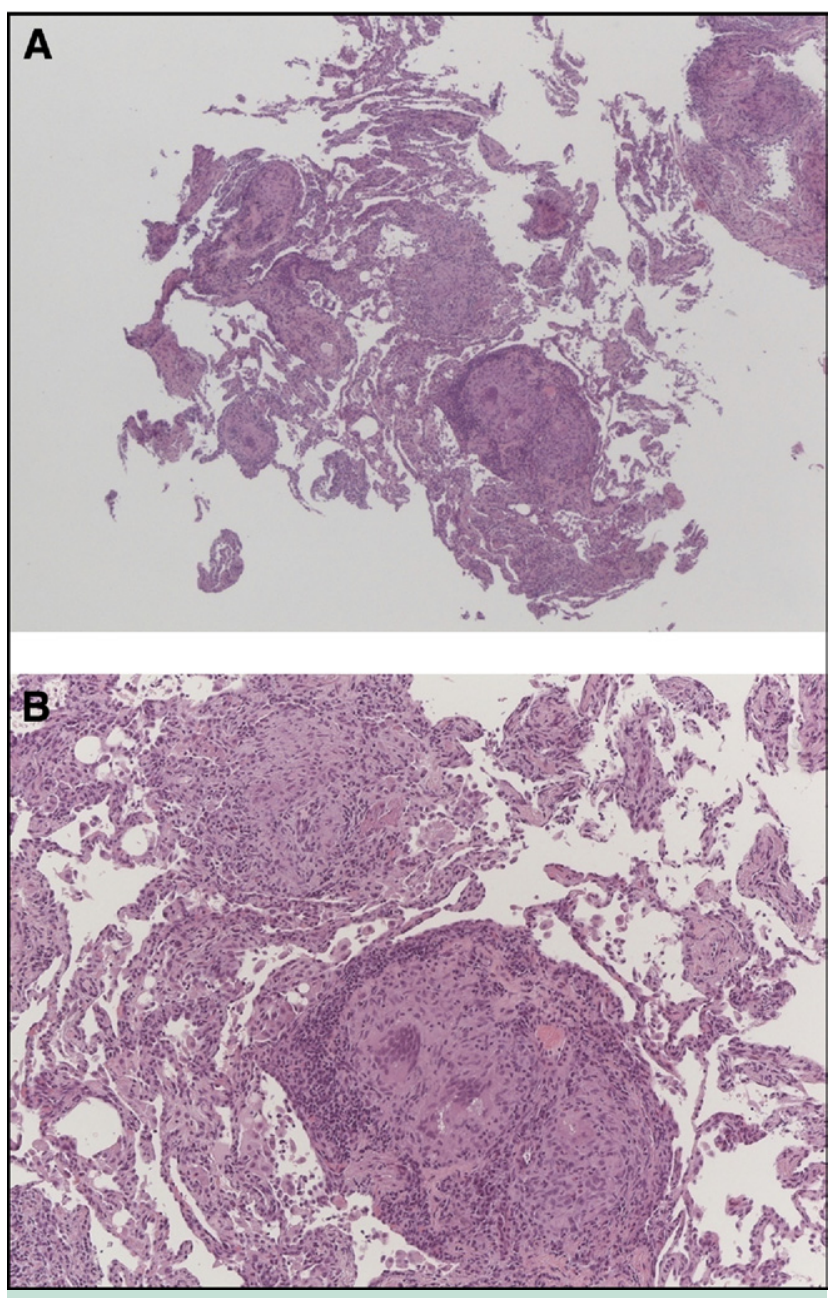

Figure 4 Transbronchial biopsy showing well-formed nonnecrotizing granulomas with lymphangitic distribution (A); higher magnification of the same biopsy (B). A. Hematoxylineosin, $40 \times$ (courtesy Dr Giulio Rossi). B. Hematoxylin-eosin, $200 \times$ (courtesy Dr Giulio Rossi)

aise, and weight loss. A chest radiograph and CT scan were performed and were essentially unremarkable and unchanged compared with previous ones, while an abdominal CT scan revealed an enlarged spleen. Serum ACE was elevated at $165 \mu \mathrm{g} / \mathrm{L}$ (normal range $0-40$ ). In addition, the patient developed severe pancytopenia and underwent bone marrow biopsy. Sadly, she deteriorated rapidly and died due to multiple organ failure. A few hours later we received the histology report of her bone marrow biopsy. The patient had an anaplastic large cell B lymphoma (Figure 5A, B, and C).

Several studies of coexistent sarcoidosis and malignancy suggest that this association is not fortuitous. ${ }^{22-24}$ Sarcoidosis may precede, follow, or occur concurrently with a number of cancers, including hematological malignanciesmostly lymphomas-and solid tumors. ${ }^{23}$ Sarcoid-like reactions occurring either adjacent to the primary malignant site or to local drainage nodes, also are common, but these should not be regarded as sarcoidosis. 


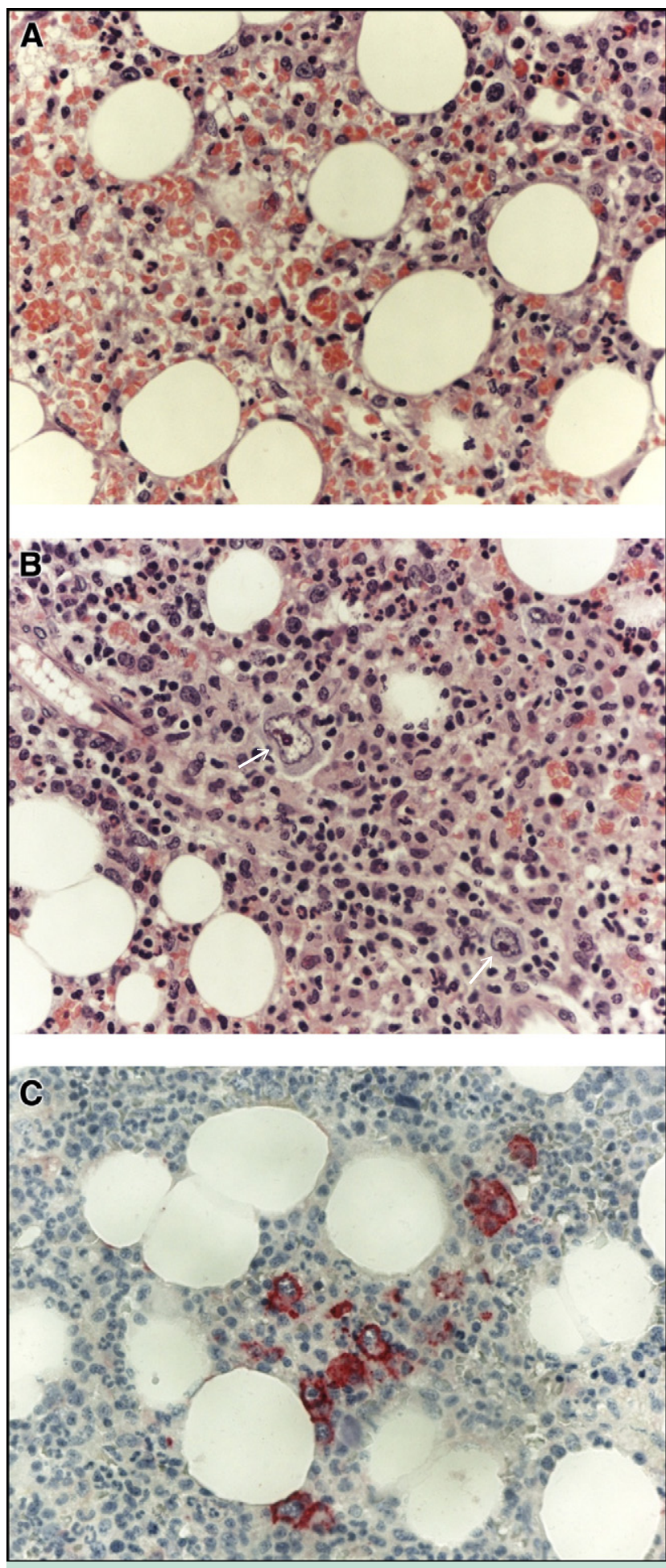

Figure 5 Bone marrow biopsy showing numerous macrophages in hemophagocytic activity (A); 2 large anaplastic cells (arrows, B); immunohistochemistry showing CD20+ malignant cells (C). A. Hematoxylin-Eosin, 400× (courtesy Dr Goretta Bonacorsi). B. Hematoxylin-Eosin, $400 \times$ (courtesy Dr Goretta Bonacorsi). C. Immunohistochemistry of the same biopsy (courtesy Dr Goretta Bonacorsi).

\section{Sarcoidosis and Oncohematologic Malignancies}

The incidence of lymphoproliferative disorders is higher among sarcoidosis patients as compared with the general population. While the precise mechanism of this association is unclear, the chronic active type of sarcoidosis appears to be responsible for an increased risk of malignant transformation of lymphoid cells. ${ }^{25}$ The term sarcoidosis-lymphoma syndrome coined by Brincker ${ }^{26}$ refers to chronic active sarcoidosis preceding the diagnosis of lymphoma, usually by several years. These patients are usually older than 40 years, and the associated lymphoma is most frequently a Hodgkin disease followed, in terms of frequency, by non-Hodgkin lymphomas. ${ }^{22,23,27}$ Persistent active sarcoidosis, anergy, and lymphopenia are usually associated with the syndrome. ${ }^{28}$ On the other hand, patients with hematologic malignancies (Hodgkin and non-Hodgkin lymphoma but also T-cell lymphoma, mycosis fungoides, chronic lymphocytic leukemia, nonlymphocytic leukemia) may subsequently develop sarcoidosis. One possibility is that sarcoidosis in these patients is triggered by the immunosuppressive treatment used for the underlying hematologic malignancy. In fact, sarcoidosis following chemotherapy has been reported in hematologic malignancies, with alpha interferon being the agent most commonly associated with development of sarcoidosis. ${ }^{29}$

\section{Sarcoidosis and Solid Tumors}

Convincing evidence suggests an increased frequency of solid tumors in patients with sarcoidosis. ${ }^{23,30}$ Specifically, organs previously affected by sarcoidosis appear to be at increased risk of developing solid tumors, likely as a consequence of the sarcoidosis-associated chronic inflammation. ${ }^{31}$ Conversely, sarcoidosis has been reported to occur following solid tumors of the cervix, liver, lung, skin (both melanoma and nonmelanoma skin cancers), testicles, and uterus, among others. ${ }^{30,32,33} \mathrm{~A}$ strong association between sarcoidosis and testicular cancer (mostly seminoma) exists: in these cases, sarcoidosis commonly follows the diagnosis of cancer, although up to one third of patients are concomitantly diagnosed with sarcoidosis and testicular cancer. When occurring concomitantly, the 2 disorders seem to follow a relatively benign course. ${ }^{34}$

\section{Sarcoid-like Reactions and Malignancies}

Sarcoid-like reactions are defined as the presence of noncaseating granulomas in patients who do not fulfill the diagnostic criteria for systemic sarcoidosis. ${ }^{23}$ In patients affected by malignancies, sarcoid-like reactions are usually observed in the lymph nodes draining the cancer. Nevertheless, they also may occur in the organ of tumor origin or at distant sites; this latter situation representing diagnostically the most challenging one. Cancer-associated sarcoid-like reactions have been observed both in patients with hematologic malignancies (chronic lymphocytic leukemia, chronic myelogenous leukemia, Hodgkin or non-Hodgkin 
disease, T-cell lymphoma, among others) and solid tumors. Whether the presence of sarcoid-like reactions correlates with better prognosis of the associated neoplasms is unclear.

Differentiating sarcoid-like reactions from systemic sarcoidosis is vital, as a wrong diagnosis of sarcoidosis and the subsequent delay in properly treating the underlying malignancy can be catastrophic. Both cancer and sarcoidosis are 18F-fluorodeoxyglucose avid; therefore, the positron emission tomography scan may be useful in selecting possible biopsy sites-by identifying organ involvement not appreciated by routine methodology - but not in differentiating between the 2 entities. If uncertainty remains, multiple biopsies may be needed in order to determine whether all fluorodeoxyglucose-avid nodes are involved with sarcoidosis or harbor cancer as well.

\section{CONCLUDING REMARKS}

The diagnosis of sarcoidosis requires the presence of a compatible clinical/radiographic picture, histologic evidence of noncaseating granulomas, and exclusion of known causes of granulomatous inflammation. Sarcoid granulomas can occur with varying rates in any organ system, but are most commonly found in the lungs and lymph nodes. Patients may be entirely asymptomatic and the condition discovered by the presence of characteristic findings on routine health screening chest radiographs $(30 \%-60 \%$ of cases) or present with a wide range of manifestations, including respiratory (dry cough, chest pain), ocular (pain, visual change), and constitutional (fever, fatigue) symptoms, or skin rashes (erythema nodosum, nodules, plaques, papules). The most common radiological features include enlarged bilateral hilar and right paratracheal lymph nodes. Frequently, sarcoidosis affects individuals aged 20 to 40 years; when it presents in persons older than 40 years, it is often associated with the development of chronic progressive disease. The acute onset of erythema nodosum or periarticular ankle inflammation, bilateral hilar lymphadenopathy (with or without fever and uveitis) - known as Löfgren syndrome-is typically associated with a benign, self-limiting disease course. ${ }^{35}$

While a positive biopsy is self-proving of diseases such as lymphoma or tuberculosis, an (isolated) biopsy is never diagnostic of sarcoidosis. Therefore, particularly in the presence of atypical manifestations (ie, unilateral hilar lymphadenopathy or isolated mediastinal lymphadenopathy without hilar lymph node enlargement) or unexpected clinical courses, histological evidence of noncaseating epithelioidcell granulomas from a single site should be considered insufficient to make the diagnosis beyond doubt because no morphological feature is pathognomonic of sarcoidosis (Table 2). One clue that sarcoidosis may not be the correct diagnosis is the patient's failure to respond to corticosteroid therapy. Another important hint concerns, among others, the appearance of the spleen on the abdominal CT scan, in the case of spleen involvement. Splenic sarcoidosis usually appears as low-attenuation nodules that may coalesce into
Table 2 Causes of Granulomatous Lung Disease

Infectious Agents

Mycobacteria (Myobacterium tuberculosis, atypical mycobacterial infection)

Fungi (histoplasmosis)

Bacteria (brucellosis, chlamydial infection)

Spirochetes (treponemal infections)

Parasites (leishmaniasis, toxoplasmosis)

Occupational/environmental exposures

Organic agents (hypersensitivity pneumonitis)

Inorganic agents (beryllium, aluminium, talc, titanium, zirconium)

Other conditions

Foreign body

Lymphomatoid granulomatosis

Cancer (lymphoma, sarcoid-like reactions)

Autoimmune disorders (Wegener granulomatosis, primary biliary cirrhosis, Churg-Strauss syndrome)

Langerhans cell histiocytosis

Bronchocentric granulomatosis

Sarcoidosis

Adapted from: Newman LS, Rose CS, Maier LA. Sarcoidosis. N Engl J Med. 1997;336:1224-1234. ${ }^{5}$

mass-like lesions. ${ }^{36}$ In our patients (both case 1 and case 2), the spleen appeared quite enlarged but without evidence of low-attenuation lesions. Furthermore, sarcoidosis is rare in the elderly (case 2).

New organ involvement from sarcoidosis is rare after $>2$ years from initial diagnosis. In addition, once the disease remits, it rarely recurs; this implies that such unusual circumstances should be suspected of having an alternative explanation. For instance, the development of a new solitary nodule (particularly in a current or ex-smoker), asymmetrical hilar, or peripheral lymphadenopathy in a patient on a previously effective treatment regimen or after a prolonged period of remission is more likely to be due to infection or neoplasm and should not be attributed to recurrence of sarcoidosis-even if the original diagnosis was histologically confirmed. ${ }^{37}$ Conversely, the presence of numerous pulmonary nodules would be more in keeping with sarcoidosis than lung cancer, although both diagnoses remain possible. In challenging instances or if malignancy is suspected, the diagnosis should be substantiated by mediastinal lymph node or open lung biopsy. In selected cases, a trial of corticosteroids may be justified to assess whether there is a clinical response consistent with sarcoidosis, bearing in mind that corticosteroids may induce a temporary/incomplete remission of lymphomas mistakenly diagnosed as systemic sarcoidosis, thus delaying the correct diagnosis and treatment.

Sarcoidosis granulomatous inflammation may develop in unusual locations, thus causing atypical patterns of organ involvement and posing a diagnostic challenge. Nevertheless, unexpected disease behaviors, such as the ones we have described, also should raise the suspicion of alternative/concomitant processes. Constitutional symptoms, he- 


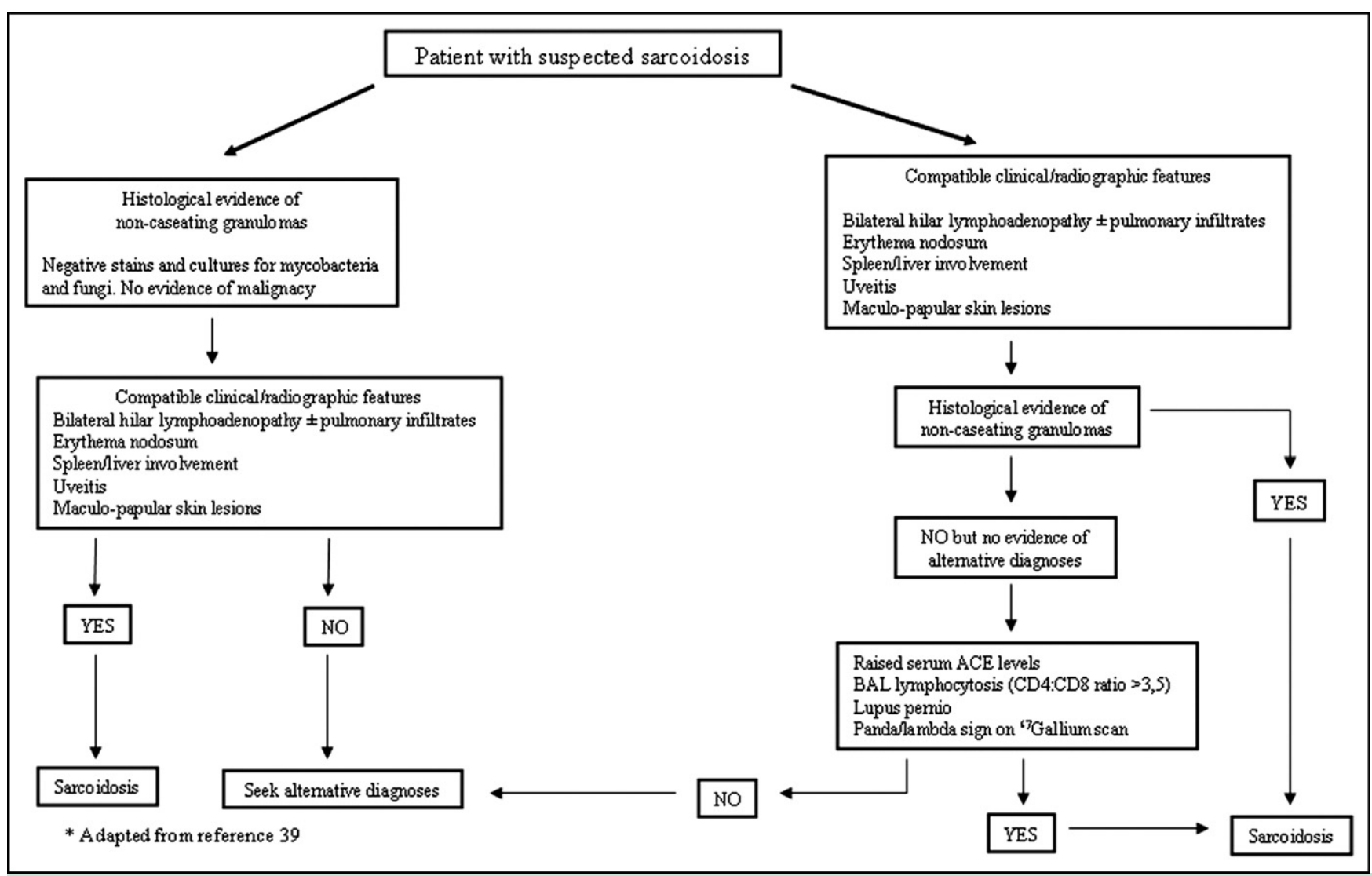

Figure 6 Diagnostic flow chart in patients with suspected sarcoidosis. Adapted from Baughman RP, Lower EE, du Bois RM. Sarcoidosis. Lancet. 2003;361:1111-1118. ${ }^{39}$

matological abnormalities, and organ involvement—such as liver, spleen, lymph nodes, and bone marrow, among others-represent nonspecific findings and do not help in differentiating sarcoidosis from neoplasm or infection. Histological confirmation of the diagnosis is therefore warranted if uncertainty persists. However, even in the presence of noncaseating granulomas, physicians should not be tempted to stop looking for known causes of granulomatous disease unless clinicoradiographic features compatible with sarcoidosis also are present. For instance, because chronic beryllium disease is pathologically and clinically indistinguishable from sarcoidosis, a beryllium lymphocyte-proliferation test looking at beryllium-specific T-lymphocyte activation should be offered to any patient evaluated for suspected sarcoidosis who has worked around metal dust or fumes.

The diagnosis of sarcoidosis may not be straightforward, and patients often report a prolonged work-up period before receiving the correct diagnosis. ${ }^{38}$ This is particularly true for isolated ocular or cutaneous disease. Conversely, combinations of typical clinical and radiographic featuresasymptomatic symmetrical bilateral hilar lymphadenopathy accompanied by erythema nodosum and ankle swellingare generally sufficient to establish a diagnosis of sarcoidosis rapidly and with little margin of error. If a clinical manifestation deviates from that expected, or defining an alternative diagnosis cannot be delayed, biopsy of the in- volved organ — possibly by sampling multiple sites—should be considered, even when there is documentation of a prior biopsy confirming an original diagnosis of sarcoidosis. Figure 6 outlines a simplified diagnostic algorithm for patients with suspected sarcoidosis.

The implications of the cases we have discussed are 3-fold:

- Sarcoidosis may precede, follow, or occur concurrently with malignancies and tuberculosis, thus representing a diagnostic challenge;

- Physicians should be ready to reconsider their initial diagnosis of sarcoidosis in the presence of atypical manifestations or persistent/progressive disease despite conventional therapy;

- Clinical acumen remains key for correct interpretation of clinical, radiological, and histological data, particularly if unusual manifestations or unexpected disease behaviors arise.

\section{References}

1. Iannuzzi MC, Rybicki BA, Teirstein AS. Sarcoidosis. $N$ Engl J Med. 2007;357:2153-2165.

2. Statement on sarcoidosis. Joint Statement of the American Thoracic Society (ATS), the European Respiratory Society (ERS) and the World Association of Sarcoidosis and other Granulomatous Disorders (WASOG) adopted by the ATS Board of Directors and by the ERS exec- 
utive committee, February 1999. Am J Respir Crit Care Med. 1999; 160:736-755.

3. Shirodaria CC, Nicholson AG, Hansell DM, et al. Lesson of the month: Necrotizing sarcoid granulomatosis with skin involvement. Histopathology. 2003;43:91-93.

4. Danila E, Zurauskas E. Diagnostic value of epithelioid cell granulomas in bronchoscopic biopsies. Intern Med. 2008;47:2121-2126.

5. Newman LS, Rose CS, Maier LA. Sarcoidosis. N Engl J Med. 1997; 336:1224-1234.

6. Lima MS, Gazzinelli G, Nascimento E, et al. Immune responses during human schistosomiasis mansoni. Evidence for antiidiotypic T lymphocyte responsiveness. J Clin Invest. 1988;78:983-988.

7. Kaplan G, Laal S, Sheftel G. The nature and kinetics of a delayed immune response to purified protein derivative of tuberculin in the skin of lepromatous leprosy patients. J Exp Med. 1988;168:1811-1824.

8. Boros DL. Experimental granulomatosis. Clin Dermatol. 1986;4:10-21.

9. Judson MA. The etiologic agent of sarcoidosis: what if there isn't one? Chest. 2003;124:6-8.

10. Burke WM, Keogh A, Maloney PJ, et al. Transmission of sarcoidosis via cardiac transplantation. Lancet. 1997;336:1579.

11. Mitchell DN, Rees RJ, Goswami KK. Transmissible agents from human sarcoid and Crohn's disease tissues. Lancet. 1976;2:761-765.

12. Kveim A. En ny og spesifikk kutan-reaksjon ved Boecks sarcoid. Nord Med. 1941;9:169-172.

13. Siltzbach LE. The Kveim test in sarcoidosis. A study of 750 patients. JAMA. 1961;178:476-482.

14. Ishioka S, Fujihara M, Takaishi M, et al. Anti-Kveim monoclonal antibody-new monoclonal antibody reacting to epithelioid cells in sarcoid granulomas. Chest. 1999;98:1255-1258.

15. Fidler HM, Rook GA, Johnson NM, McFadden J. Mycobacterium tuberculosis DNA in tissue affected by sarcoidosis. BMJ. 1993;306: 546-549.

16. Fité E, Fernández-Figueras MT, Prats R, et al. High prevalence of Mycobacterium tuberculosis DNA in biopsies from sarcoidosis patients from Catalonia, Spain. Respiration. 2006;73:20-26.

17. Drake WP, Pei Z, Pride DT, et al. Molecular analysis of sarcoidosis tissues for mycobacterium species DNA. Emerg Infect Dis. 2002;8: 1334-1341.

18. Song Z, Marzilli L, Greenlee BM, et al. Mycobacterial catalaseperoxidase is a tissue antigen and target of the adaptive immune response in systemic sarcoidosis. J Exp Med. 2005;201:755-767.

19. Drake WP, Dhason MS, Nadaf M, et al. Cellular recognition of Mycobacterium tuberculosis ESAT-6 and KatG peptides in systemic sarcoidosis. Infect Immun. 2007;75:527-530.
20. Chen ES, Wahlström J, Song Z, et al. T cell responses to mycobacterial catalase-peroxidase profile: a pathogenic antigen in systemic sarcoidosis. J Immunol. 2008;181:8784-8796.

21. Spagnolo P, du Bois RM. Genetics of sarcoidosis. Clin Dermatol. 2007:25:242-249.

22. Caras WE, Dillard T, Baker T, Pluss J. Coexistence of sarcoidosis and malignancy. South Med J. 2003;96:918-922.

23. Cohen PR, Kurzrock R. Sarcoidosis and malignancy. Clin Dermatol. 2007:25:326-333

24. Seersholm N, Vestbo J, Viskum K. Risk of malignant neoplasms in patients with pulmonary sarcoidosis. Thorax. 1997;52:892-894.

25. Brincker H. Coexistence of sarcoidosis and malignant disease: causality or coincidence? Sarcoidosis. 1989;6:31-43.

26. Brincker H. The sarcoidosis-lymphoma syndrome. Br J Cancer. 1986; 54:467-473.

27. Karakantza M, Matutes E, MacLennan K, et al. Association between sarcoidosis and lymphoma revisited. J Clin Pathol. 1996;49: 208-212.

28. Suen JS, Forse MS, Hyland RH, Chan CK. The malignancy-sarcoidosis syndrome. Chest. 1990;98:1300-1302.

29. Shigemitsu H. Is sarcoidosis frequent in patients with cancer? Curr Opin Pulm Med. 2008;14:478-480.

30. Brincker H. Solid tumors preceding or following sarcoidosis. Med Pediatr Oncol. 1987;15:82-88.

31. Askling J, Grunewald J, Eklund A, Hillerdal G, Ekbom A. Increased risk for cancer following sarcoidosis. Am J Respir Crit Care Med. 1999;160:1668-1672.

32. Brincker H. Sarcoidosis and malignancy. Chest. 1995;108:1472-1474.

33. Reich JM. Malignant neoplasms in pulmonary sarcoidosis. Thorax. 1998;53:625-626

34. Paparel P, Devonec M, Perrin P, et al. Association between sarcoidosis and testicular carcinoma: a diagnostic pitfall. Sarcoidosis Vasc Diffuse Lung Dis. 2007;24:95-101.

35. Mañá J, Gómez-Vaquero C, Montero A, et al. Löfgren's syndrome revisited: a study of 186 patients. Am J Med. 1999;107:240-245.

36. Judson MA. The diagnosis of sarcoidosis. Clin Chest Med. 2008;29: 415-427.

37. Moller DR. Rare manifestations of sarcoidosis. Eur Respir Mon. 2005; 32:233-250

38. Judson MA, Thompson BW, Rabin DL et al; ACCESS Research Group. The diagnostic pathway to sarcoidosis. Chest. 2003;123:406412.

39. Baughman RP, Lower EE, du Bois RM. Sarcoidosis. Lancet. 2003; 361:1111-1118 